\title{
VULCANISMO DE AFINIDAD ADAQUÍTICA EN EL MIEMBRO INFERIOR DE LA FORMACIÓN COMBIA (MIOCENO TARDÍO) AL SUR DE LA SUBCUENCA DE AMAGA, NOROCCIDENTE DE COLOMBIA
}

\author{
Carlos Borrero $^{1}$; Luz Mary Toro-Toro ${ }^{1}$
}

DOI: http://dx.doi.org/10.18273/revbol.v38n1-2016005 (c) () ()

Forma de citar: Borrero, C., y Toro-Toro, L.M. 2016. Vulcanismo de afinidad adaquítica en el miembro inferior de la Formación Combia (Mioceno tardío) al sur de la subcuenca de Amaga, noroccidente de Colombia. Boletín de Geología, 38(1): 87-100.

\section{RESUMEN}

En este trabajo se presenta la química de los depósitos volcaniclásticos del Miembro Inferior de la Formación Combia y los cuerpos sub-volcánicos que la intruyen, aflorantes en la parte sur de la subcuenca de Amagá, en el valle interandino del río Cauca, Colombia. Corresponden a una suite andesítica-dacítica calcoalcalina de medio K con contenido de $\mathrm{SiO}_{2}$ entre 59,04 y 62,86\%, y solo una muestra corresponde a un basalto toleítico $\left(\mathrm{SiO}_{2}=50,6 \%\right)$. Las muestras se identifican por tener altas proporciones de LILE/HFSE, Ba/LREE, Sr/Nd, y empobrecimiento relativo en $\mathrm{Nb}$ y Ta, típicas de magmas de una zona de subducción. El diagrama multielemental de tierras raras indica que dos muestras tienen relaciones diferentes de MREE/HREE $(\mathrm{Gd} / \mathrm{Yb})>3,9$ y de LREE/HREE $(\mathrm{La} / \mathrm{Yb})>19,7$ que las separa del resto de las muestras. La mayoría de las muestras presentan firmas adaquíticas caracterizadas por: $\mathrm{SiO}_{2}>56 \%, \mathrm{Al}_{2} \mathrm{O}_{3}>15 \%, \mathrm{MgO}<3 \%, \mathrm{Na}_{2} \mathrm{O}$ entre 3,5 y $7,5 \%, \mathrm{~K}_{2} \mathrm{O} / \mathrm{Na}_{2} \mathrm{O} \sim 0.42$, alto fraccionamiento de HREE, ausencia de anomalías de $\mathrm{Eu}, \mathrm{Yb} \leq 1,8 \mathrm{ppm}, \mathrm{Y}<18 \mathrm{ppm}$, y altos contenidos de $\mathrm{Sr}(>400 \mathrm{ppm}), \mathrm{Sr} / \mathrm{Y}>20$ y La/Yb $>20$. Los resultados de isótopos radiogénicos para cuatro muestras de los cuerpos sub-volcánicos muestran valores muy cercanos: ${ }^{87} \mathrm{Sr} /{ }^{86} \mathrm{Sr}=0,704201-0,704293$ y ${ }^{143} \mathrm{Nd} /{ }^{144} \mathrm{Nd}=0,512849-0,512907$, los cuales sugieren una fuente manto derivada, previamente metasomatizada con fluidos del slab, sin descartar la fusión parcial de la misma, con generación de los magmas adaquíticos en niveles profundos de la corteza. Las rocas y depósitos aquí descritos corresponden a un segmento del arco magmático calcoalcalino de edad Mioceno que se extiende en el norte de los Andes. En el Miembro Inferior de La Formación Combia, en el sur de la subcuenca de Amagá, se debería incluir los cuerpos sub-volcánicos que la intruyen como una suite de afinidad adaquítica.

Palabras clave: Formación Combia, magmatismo de arco, adaquitas, manto derivado, noroeste de Colombia

\section{ADAKITE-LIKE SIGNATURE IN THE LOWER MEMBER OF THE COMBIA FORMATION (LATE MIOCENE) AT THE SOUTHERN OF AMAGÁ SUB-BASIN, NORTHWEST COLOMBIA}

\begin{abstract}
In this study, we report chemistry of samples of the volcaniclastic deposits from the Lower Member of the Combia Formation and the intruding sub-volcanic bodies. These rocks are located at the southern of Amagá sub-basin of the Cauca River, Colombia. Most of the samples correspond to a medium-K calc-alkaline andesitic-dacitic suite $\left(\mathrm{SiO}_{2}=59\right.$, $04-62,86$ wt. \%), and only one is a toleiitic basalt $\mathrm{SiO}_{2}=50,6 \mathrm{wt} \%$. The samples have high LILE/HFSE, Ba/LREE, Sr/ $\mathrm{Nd}$ ratios and $\mathrm{Nb}$ and Ta relative depletion, typical of magmas in a subduction system. The REE patterns show that two samples have high MREE/HREE and LREE/HREE ratios $(\mathrm{Gd} / \mathrm{Yb}>3,9$ and $\mathrm{La} / \mathrm{Yb}>19,7)$ in comparison with the rest of the samples. Most of the samples have an adakite-like signature characterized by $\mathrm{SiO}_{2}>56 \mathrm{wt} \%, \mathrm{Al}_{2} \mathrm{O}_{3}>15 \mathrm{wt} \%$, $\mathrm{MgO}<3 \mathrm{wt} \%, \mathrm{Na}_{2} \mathrm{O}$ between 3,5 to $7,5 \mathrm{wt} \%, \mathrm{~K}_{2} \mathrm{O} / \mathrm{Na}_{2} \mathrm{O} \sim 0.42$, high HREE fractionation, lack of Eu anomalies , $\mathrm{Yb}$ $\leq 1,8 \mathrm{ppm}, \mathrm{Y}<18 \mathrm{ppm}$, high Sr contents $(>400 \mathrm{ppm}), \mathrm{Sr} / \mathrm{Y}>20$ and $\mathrm{La} / \mathrm{Yb}>20$. Variations of the radiogenic isotopes from four sub- volcanic bodies are small and their values: ${ }^{87} \mathrm{Sr} /{ }^{86} \mathrm{Sr}=0,704201-0,704293 ;{ }^{143} \mathrm{Nd} /{ }^{144} \mathrm{Nd}=0,512849$ 0,512907 are indicative of melts from a mantle-derived source, although an interaction with slab melts is not discarded, and the adakitic magmas were generated at the base of the crust. The volcanic sequence described in this study is a segment of the Miocene magmatic arc extending along the northern Andes. According to our results, in the Lower Member of the Combia Formation, at the southernmost Amagá sub-basin sector, it must be included the intruding subvolcanic bodies as an adakitic suite.
\end{abstract}

Keywords: Combia Formation, magmatic arc, adakites, mantle-derived, northwestern Colombia

${ }^{1}$ Departamento de Ciencias Geológicas, Universidad de Caldas. Colombia, borrero_c@yahoo.com, luz.toro@ucaldas.edu.co 


\section{INTRODUCCIÓN}

La Formación Combia es una unidad que se extiende a lo largo de la parte media y alta del valle interandino del río Cauca entre las Cordilleras Central y Occidental en el noroeste de Colombia, denominada inicialmente por Grosse (1926) como "Estratos de Combia" y redefinida estratigráficamente por González (1980) quien la dividió en dos miembros: Inferior o Volcánico y el Superior o Volcaniclástico.

El Miembro Inferior está compuesto por lavas basálticas y andesíticas, aglomerados, brechas volcánicas, tobas, conglomerados con matriz tobácea y areniscas tobáceas, y se incluyen cuerpos de pórfidos andesíticos y dacíticos (Restrepo et al., 1981; Leal-Mejía, 2011). Los productos extrusivos e intrusivos muestran una afinidad geoquímica mezclada entre toleítica y calcoalcalina (e.g. Jaramillo, 1976; Tejada et al., 2007; Leal-Mejía, 2011). El Miembro Superior está constituido esencialmente por niveles gruesos de conglomerados con intercalaciones de areniscas de grano medio a fino con alto contenido de material volcánico y arcillolitas (Grosse, 1926; González, 1980; López et al., 2006).

Entre los departamentos de Caldas y Risaralda, en la parte media del valle del río Cauca, y que corresponde geológicamente a la parte sur de la subcuenca de Amagá, se localiza una cuña de depósitos volcaniclásticos del Miembro Inferior, la cual es intruida en los sectores oriental y central de esta subcuenca por una serie de cuerpos sub-volcánicos porfiríticos de composición andesítica y dacítica que forman espinas, cuellos volcánicos y domos denominados de manera genérica como "pórfidos" (e.g. Tejada et al., 2007). Ambas unidades volcánicas con edades combinadas entre 12 y 6 Ma (Restrepo et al., 1981; Ordóñez y Pimentel, 2001; López et al., 2006, Ramírez et al., 2006; Leal-Mejía, 2011) constituyen el registro del arco volcánico del Mioceno tardío en el noroccidente de Colombia, el cual estuvo relacionado con la interacción de la Placa Nazca y la parte noroccidental del continente suramericano (Cediel et al., 2011).

En este trabajo se revisa la química de elementos mayores y elementos traza de los depósitos volcaniclásticos del Miembro Inferior de la Formación Combia y de los cuerpos sub-volcánicos que la intruyen, así como las razones isotópicas de $\mathrm{Sr}$ y $\mathrm{Nd}$ de los cuerpos subvolcánicos aflorantes en la parte sur de la subcuenca de Amagá en los departamentos de Caldas y Risaralda (FIGURA 1). Los resultados muestran la posibilidad de la existencia de un vulcanismo adaquítico en el Mioceno tardío en la parte sur de esta subcuenca y se sugiere que podría estar relacionado con fundidos derivados del manto que evolucionaron en la parte más profunda de la corteza, pero no se descarta la posibilidad de que tengan también alguna mezcla con fundidos de la placa subducente.

Este arco volcánico que se localiza en el centrooccidente de Colombia, se enmarcaría en el Cinturón Metalogénico del Cauca Medio de edad Mioceno, según el esquema general de Sillitoe (2008), y se puede integrar a los arcos con vulcanismos similares en el centro y suroccidente de Colombia y norte de Ecuador, los cuales formaron un solo cinturón magmático, aunque fragmentado en el Mioceno (Schütte, 2010; Leal-Mejía, 2011, Cediel et al., 2011).

\section{MARCO GEOLÓGICO}

En la FIGURA 1 se muestra el mapa geológico del área de trabajo entre aproximadamente $5^{\circ}$ y $5^{\circ} 40^{\prime}$ Lat. Norte y $75^{\circ} 25^{\prime}$ y $75^{\circ} 50^{\prime}$ Long W, modificado de Gómez et al. (2007), y corresponde a la parte sureste de la subcuenca de Amagá, que es la parte más meridional de la cuenca Amagá-Cauca-Patía (ACP, Sierra and Marín, 2011) y el borde occidental de la Cordillera Central. El basamento de la Cordillera Central en esta área está constituido por los complejos del Cretácico inferior: Arquía y Quebradagrande (Moreno et al., 2008). En el sector central y occidental del área (FIGURA 1), la subcuenca de Amagá tiene un basamento constituido por la Formación Barroso descrita por Álvarez (1971) que continúa en la Cordillera Occidental; el relleno sedimentario y volcaniclástico en esta zona está constituido por el Miembro Superior de la Formación Amagá (cf. Ramírez et al. 2006), la Formación Combia y agrupaciones de cuerpos sub-volcánicos que tienen dimensiones variadas.

El sistema de fallas de Romeral define los límites en los diferentes complejos que forman el basamento de la Cordillera Central, es un sistema de rumbo dextro-lateral con un componente inverso e incluye las fallas de San Jerónimo, Silvia-Pijao y Cauca-Almaguer (FIGURA 1). La falla más occidental de este sistema, Cauca-Almaguer es el límite oriental entre la subcuenca de Amagá con la Cordillera Central (cf. Gómez et al., 2007). En el oeste, la sub-cuenca de Amagá está separada de la Cordillera Occidental por el sistema de fallas del Cauca que tiene vergencia hacia el este; la falla de Sevilla (ver FIGURA 1 parte inferior izquierda) es el trazo más oriental de este sistema (Gómez et al., 2007). 


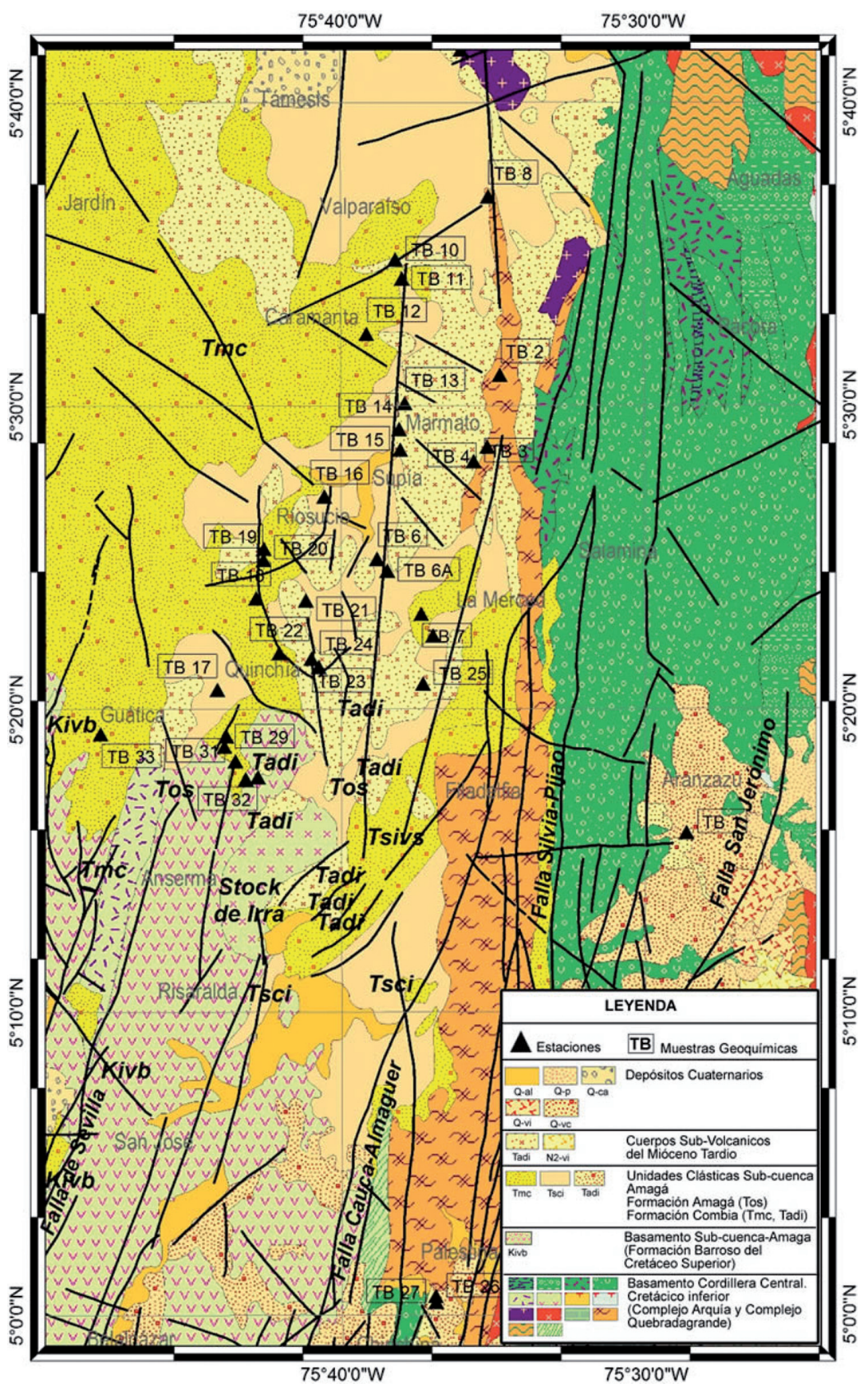

FIGURA 1. Mapa geológico del área de estudio, modificado de Gómez et al. (2007). Triángulos negros muestran la localización de las estaciones del trabajo de campo, y las muestras con geoquímica se muestran resaltadas en un rectángulo con su numeración igual a la TABLA 1 .

\section{Formación Combia}

La Formación Combia aflora en el noroeste Colombiano, en la subcuenca Amagá, en los departamentos de Antioquia, Risaralda y Caldas, y está dividida en dos miembros: El Miembro Inferior de carácter volcánico y el Miembro Superior de carácter volcaniclástico (González, 1980). El Miembro Inferior (FIGURA 1) ocupa la mayor parte del sector central y norte del área trabajada, con escasos afloramientos de flujos de lavas de composición basáltica, andesita basáltica y andesítica reportadas en el suroeste del departamento de Antioquia (e.g. López et al., 2006; Tejada et al., 2007), y afloran principalmente los depósitos de flujos piroclásticos de bloques y cenizas, formando secuencias de hasta $200 \mathrm{~m}$ de espesor en superficie (FIGURA 2A y C). 

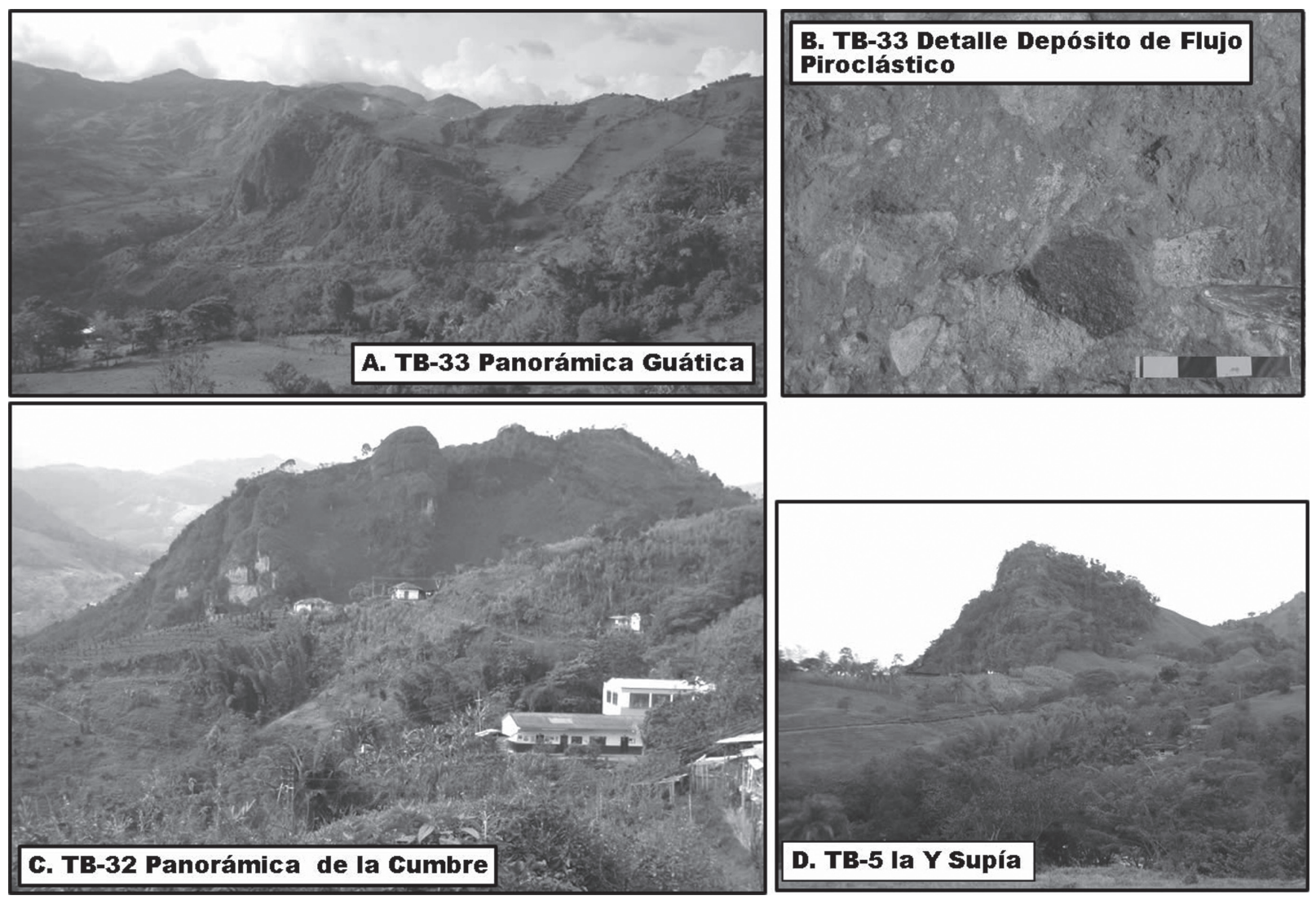

FIGURA 2. A. Estación TB-33, panorámica del área del municipio de Guática (departamento de Risaralda) mostrando el espesor de los depósitos de flujos piroclásticos del Miembro Inferior de la Formación Combia. B. detalle de los depósitos de flujos piroclásticos de bloques y cenizas de la estación TB-33; escala, barra en $\mathrm{cm}$. C. Estación TB-32, panorámica del sector de La Cumbre en el municipio de Quinchía (departamento de Risaralda) mostrando el espesor de los depósitos de flujos piroclásticos del Miembro Inferior de la Formación Combia. D. Estación TB-5, sector de la Y, municipio de Supía (departamento de Caldas): depósitos del Miembro Superior de la Formación Combia intruidos por un domo volcánico. Localización de las estaciones en la FIGURA 1.

Los depósitos de flujos piroclásticos en promedio tienen una relación fragmentos: matriz de 70-80\%: 30-20\%; los fragmentos corresponden a bloques y bombas, angulares a sub-redondeados de composición andesítica y dacítica con texturas porfiríticas (FIGURA 2B) y con variable grado de vesiculación; los colores de los fragmento varían de grises a verdes y cafés. La matriz de los depósitos es de composición vitro-cristalo-lítica, de color gris claro, con tamaño de grano de ceniza media a gruesa; los depósitos casi siempre forman una sola unidad de enfriamiento por presentar un soldamiento intermedio extensivo (sensu Murcia et al., 2013). Los eventos de flujos piroclásticos se relacionan con la destrucción de parte de los cuerpos sub-volcánicos descritos más adelante.

El Miembro Superior o volcaniclástico de la Formación Combia está compuesto por rocas sedimentarias con aporte de material volcánico, de composición similar al que constituye el Miembro Inferior y aflora en la parte central del área trabajada (FIGURA 1).

\section{Cuerpos sub-volcánicos}

Los cuerpos sub-volcánicos se localizan de manera aislada o formando grupos con dimensiones variables. En el área de trabajo estos cuerpos se ubican principalmente en el oriente, límite entre la subcuenca de Amagá y la Cordillera Central (FIGURA 1), alineándose de manera general con la dirección del valle actual del río Cauca.

Los diferentes cuerpos sub-volcánicos llamados comúnmente "pórfidos", se identifican por sus geomorfologías positivas (FIGURA 3A, B, E). Estos cuerpos se encuentran intruyendo a los depósitos piroclásticos del Miembro Inferior y a las rocas sedimentarias del Miembro Superior (FIGURA 2D). De acuerdo con Patarroyo (2014), la mayoría de estos cuerpos tienen textura porfirítica de grano 
grueso, cristales de anfíbol y plagioclasa con tamaño centimétrico en una matriz afanítica (FIGURA 3C, F). Microscópicamente las rocas están constituidas por fenocristales entre un 10 y $25 \%$, principalmente de plagioclasa y hornblenda, y en muy baja proporción granates, que suelen estar englobados en una masa fundamental cristalo-vítrea con desarrollo de una textura felsítica. Los fenocristales de plagioclasa presentan tamaños entre 1 y $3 \mathrm{~mm}$, y con formas euhedrales a subhedrales, a menudo con zonamiento oscilatorio; también se observaron microlitos de plagioclasa. El anfíbol oxi-hornblenda se presenta como fenocristal con tamaños variables, en general menores a $1 \mathrm{~mm}$. La masa fundamental está constituida por cristales submilimétricos de anfíboles y agregados anhedrales de cuarzo y plagioclasa; además se presentan sectores con masas irregulares de carbonatos. No se observaron xenolitos en las muestras analizadas.

Los cuerpos sub-volcánicos son coetáneos con el sistema de rocas extrusivas de la Formación Combia con edades entre 12-6 Ma (K/Ar en hornblendas: Restrepo et al., 1981; trazas de fisión en circones: Ramírez et al., 2006; $\mathrm{K} / \mathrm{Ar}$ en roca total: Leal-Mejía, 2011).
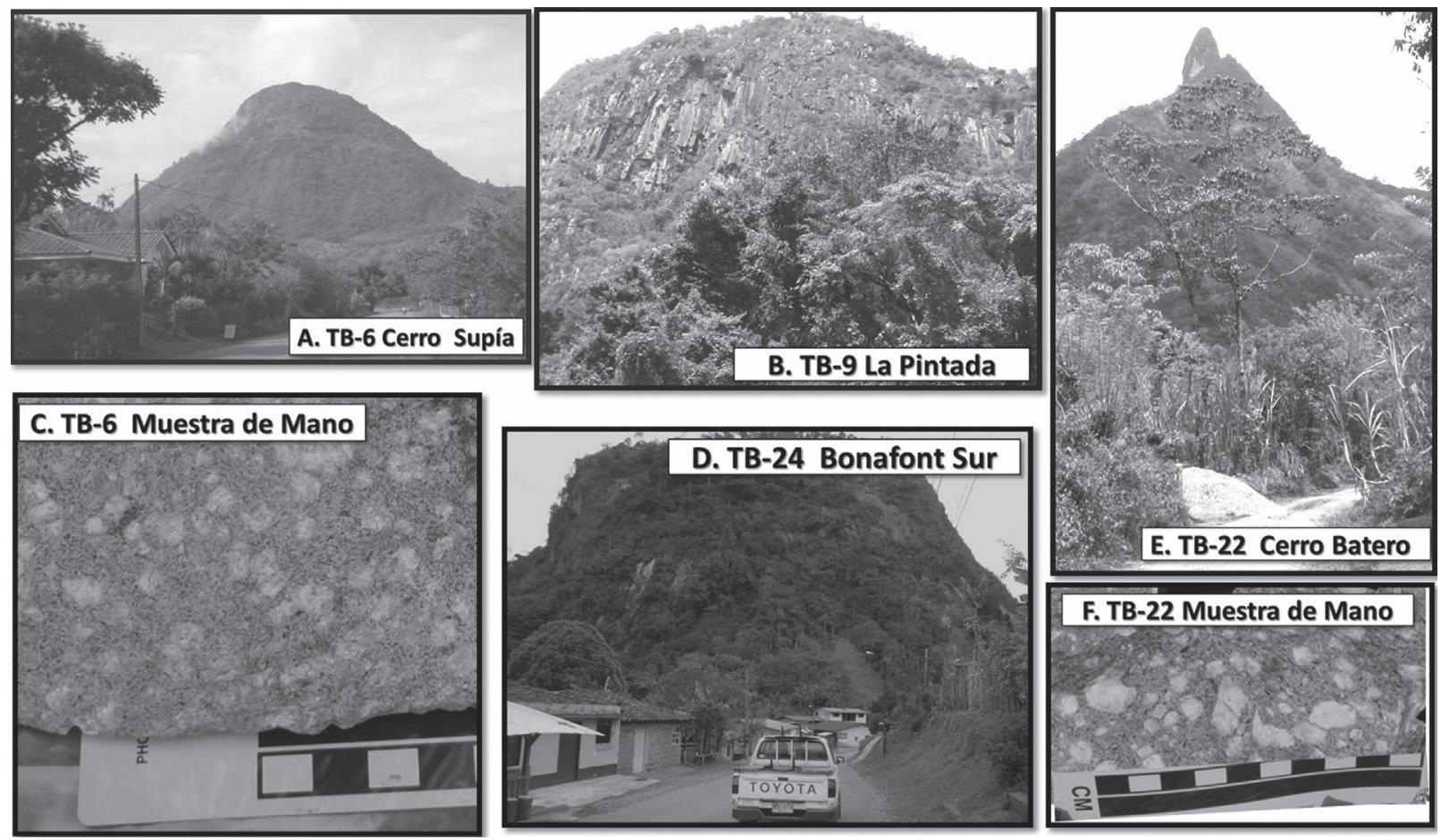

FIGURA 3. A. Estación TB-6, domo volcánico, cerro Supía, al SE del municipio de Supía (depto. de Caldas). B. Estación TB-9, cuello volcánico, sur del sector de La Pintada, límite Caldas-Antioquia. C. Muestra de mano de las rocas porfiríticas del domo de la Estación TB-6, escala en cm. D. Estación TB-24, domo galleta al sur del corregimiento de Bonafont (municipio de Riosucio, depto. de Caldas). E. Estación TB-22, domo y espina volcánica del cerro Batero, al NW del corregimiento de Bonafont (municipio de Riosucio, depto. de Caldas). F. Detalle del tamaño de los fenocristales en una muestra de mano del domo del Cerro Batero.

\section{MATERIALES Y MÉTODOS}

Para estudiar la composición química y elucidar los procesos de formación magmática se escogieron 12 muestras para análisis químico en roca total (ver FIGURA 1 para localización de las muestras y TABLA 1 para resultados de los análisis). Una muestra corresponde a una lava basáltica (TB-10), dos muestras pertenecen a fragmentos de depósitos piroclásticos de bloques y cenizas en el área de Quinchía (TB-30 y TB-31), y ocho a los cuerpos sub-volcánicos del Miembro Inferior de la
Formación Combia (TB-4, TB-6, TB-9, TB-14, TB-17, TB-20, TB-21 y TB-22). Adicionalmente, para verificar si la actividad magmática con igual afinidad geoquímica continúa por fuera de la sub-cuenca de Amagá, se escogió la muestra TB-26 que pertenece a un cuerpo sub-volcánico aflorante (parte inferior de la FIGURA 1) en el sector de Palestina (departamento de Caldas).

Las determinaciones de elementos mayores y trazas en roca total fueron realizadas en Acme Analytical Laboratories Colombia (TABLA 1). Fueron tomados 0,2 
$\mathrm{g}$ de cada muestra triturada y mezclada con un fundente $\mathrm{LiBO}_{2} / \mathrm{Li}_{2} \mathrm{~B}_{4} \mathrm{O}_{7}$ para fundirla en una mufla. La perla enfriada es disuelta en $\mathrm{HNO}_{3}$ al 5\%, y son analizadas por ICP y/o ICP-MS. La pérdida por ignición (LOI) se halló por la diferencia de peso después de la ignición a $1.000^{\circ}$ C. Los límites de detección para elementos mayores incluido LOI fueron de $0,01 \%$ con excepción de $\mathrm{Fe}_{2} \mathrm{O}_{3}$ y $\mathrm{K}_{2} \mathrm{O}: 0,04 \%$. Para elementos traza y tierras raras los límites de detección fueron $5 \mathrm{ppb}(\mathrm{Au}) ; 0,05 \mathrm{ppm}(\mathrm{Sm}$, Gd, Dy, Yb); 0,01 ppm (Tb, Tm, Lu, Ni); 0,1 ppm (La, $\mathrm{Ce}, \mathrm{Cs}, \mathrm{Rb}, \mathrm{U}, \mathrm{Nb}, \mathrm{Ta}, \mathrm{Pb}, \mathrm{Y}, \mathrm{Cu}$ ); 0,2 ppm (Pr, Eu, Ho, Th, Co); 0,3 ppm (Nd, Er, Sr); 1 ppm (Zn); y 5 ppm (Ba).

Para estudiar la composición isotópica de $\mathrm{Sr}-\mathrm{Nd}$ se llevaron a cabo cuatro análisis en roca total de las muestras TB-6, TB-9, TB-17 y TB-22 de los cuerpos sub-volcánicos (TABLA 2) y fueron llevados a cabo en un (MC)-ICP-MS multi-colector Thermo-Finnigan Neptune en Washington State University (Pullman, WA). Para los análisis isotópicos de roca total de $\mathrm{Sr}$ y Nd, se tomaron alícuotas de polvos de roca total de aproximadamente 0,25 gramos, que fueron disueltas a alta presión en bombas de teflón encamisadas en acero con una mezcla $10: 1$ de $\mathrm{HF}$ y $\mathrm{HNO}_{3}$ a $150^{\circ} \mathrm{C}$ entre 5 y 7 días. Los análisis de $\mathrm{Sr}$ fueron corregidos por fraccionamiento de masas usando ${ }^{86} \mathrm{Sr} /{ }^{87} \mathrm{Sr}=0,1194$ y normalizados usando NBS-987 como estándar. Análisis de $\mathrm{Nd}$ fueron corregidos por fraccionamiento de masas usando ${ }^{146} \mathrm{Nd} /{ }^{144} \mathrm{Nd}=0,7219$ y normalizados usando el estándar Ames Nd. Las incertidumbres de las composiciones isotópicas reflejan errores $2 \sigma$ que ocurren durante la corrida de las muestras en el laboratorio.

\section{RESULTADOS}

\section{Geoquímica}

Los análisis químicos de elementos mayores en roca total muestran una estrecha composición para la mayoría de las muestras, las cuales varían entre andesitas y dacitas $\left(\mathrm{SiO}_{2}\right.$ entre 59,04 y $62,86 \%$ ) y se localizan en el campo subalcalino del diagrama TAS (FIGURA 4A) y calcoalcalino del diagrama AFM (FIGURA 4B), con la sola excepción de una muestra del Miembro Inferior que presenta composición basáltica ( $\mathrm{SiO}_{2}$ 50,60 \%) (FIGURA 4A) y se ubica en el campo toleítico (FIGURA 4B). El LOI de las muestras varía entre 1,1 y $3,7 \%$, con siete muestras con menos del $2 \%$. Los valores más altos, entre 2,8 y $3,7 \%$, corresponden a las muestras de los depósitos de flujos piroclásticos (TB-30 y TB-31) que están medianamente alteradas por estar localizadas en el cinturón metalogénico de Quinchía (departamento de Risaralda), mientras que dos muestras de los cuerpos sub-volcánicos (TB-4 y TB14) y una (TB-10) que corresponde al basalto presentan valores intermedios de LOI entre 2,4 y 2,6\%. Muestras con LOI $>2 \%$ fueron tratadas con precaución para la interpretación de los resultados. Los valores de $\mathrm{K}_{2} \mathrm{O}$ permiten ubicar la mayoría de muestras, que tienen $\mathrm{LOI}<$ $2,6 \%$, en el campo de las series calcoalcalinas de potasio medio (Gill, 1981).

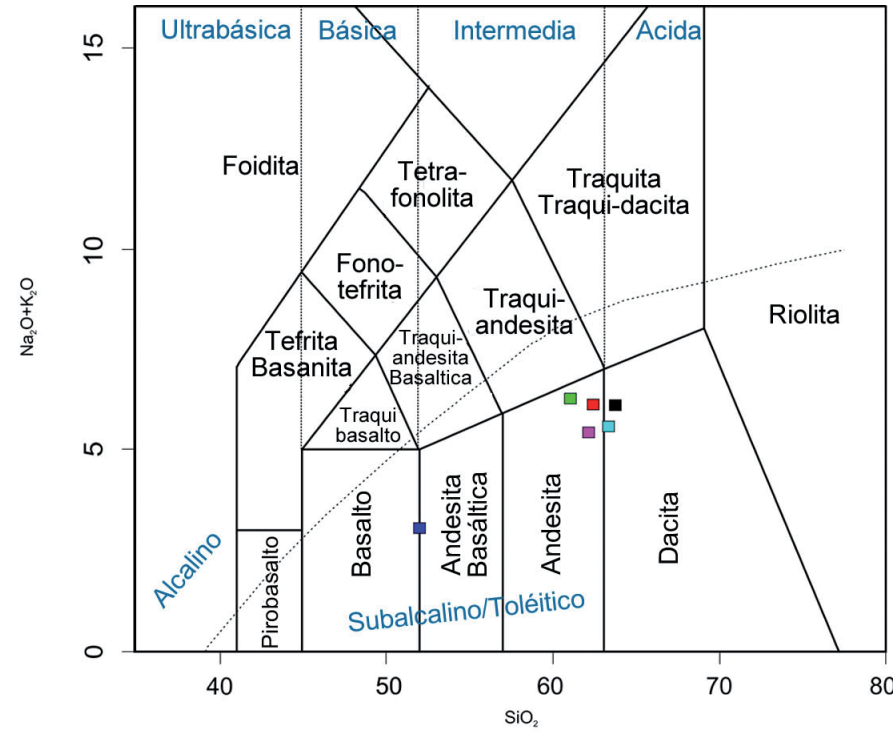

A.

FIGURA 4. A. Diagrama TAS (LeBas et al., 1986) con la ubicación de las muestras, muchas de ellas coinciden en su posición. B. Diagrama AFM (Irvine and Baragar, 1971) para diferenciar series toleíticas de las alcalinas. En las series calco-alcalinas también hay coincidencia de ubicación de las muestras. En la leyenda, con el número de la muestra entre paréntesis se muestra el valor del \% de LOI. Localización de las muestras en la FIGURA 1. 
TABLA 1. Geoquímica de elementos mayores, tierras raras y traza de las muestras del sur de la subcuenca de Amagá, localización muestras en la FIGURA 1.

\begin{tabular}{|c|c|c|c|c|c|c|c|c|c|c|c|c|}
\hline Muestra & TB-4 & TB-6 & TB-9 & TB-10 & TB-14A & TB-17 & TB-20 & TB-21 & TB-22 & TB-26 & TB-30 & TB-31 \\
\hline \multirow{2}{*}{ Localización } & N5 28.634 & N5 24.950 & N5 41.761 & N5 34.822 & N5 29.236 & N5 20.629 & N5 25.293 & N5 23.580 & N5 21.844 & N5 00.622 & N5 17.687 & N5 18.285 \\
\hline & W75 35.177 & W75 38.812 & W75 36.021 & W75 38.021 & W75 38.078 & W75 44.076 & W75 42.540 & W75 41.165 & W75 42.033 & W75 36.927 & W75 43.145 & W75 43.490 \\
\hline $\mathrm{SiO}_{2}$ & 61,69 & 61,17 & 60,08 & 50,60 & 61,33 & 61,04 & 59,66 & 59,84 & 59,95 & 62,86 & 60,45 & 59,04 \\
\hline $\mathrm{Al}_{2} \mathrm{O}_{3}$ & 16,52 & 16,65 & 17,32 & 18,70 & 16,69 & 16,13 & 16,92 & 16,90 & 16,06 & 16,78 & 17,01 & 18,01 \\
\hline $\mathrm{Fe}_{2} \mathrm{O}_{3}$ & 5,31 & 5,71 & 5,98 & 9,93 & 5,91 & 6,54 & 6,81 & 5,94 & 6,71 & 5,13 & 5,01 & 6,78 \\
\hline MgO & 1,74 & 1,89 & 2,11 & 3,53 & 1,74 & 2,12 & 2,28 & 2,42 & 2,63 & 1,82 & 2,32 & 1,66 \\
\hline $\mathrm{CaO}$ & 5,28 & 5,58 & 6,17 & 10,30 & 5,18 & 6,06 & 6,11 & 6,16 & 6,08 & 5,14 & 4,36 & 4,43 \\
\hline $\mathrm{Na}_{2} \mathrm{O}$ & 3,86 & 3,79 & 4,34 & 2,61 & 3,99 & 3,50 & 3,83 & 3,86 & 3,66 & 3,29 & 2,42 & 3,32 \\
\hline $\mathbf{K}_{2} \mathbf{O}$ & 1,98 & 2,07 & 1,64 & 0,25 & 1,28 & 1,73 & 1,80 & 1,96 & 2,06 & 2,00 & 4,04 & 1,88 \\
\hline $\mathrm{TiO}_{2}$ & 0,56 & 0,56 & 0,65 & 0,94 & 0,56 & 0,62 & 0,62 & 0,63 & 0,65 & 0,49 & 0,56 & 0,58 \\
\hline $\mathbf{P}_{2} \mathbf{O}_{5}$ & 0,28 & 0,29 & 0,21 & 0,31 & 0,30 & 0,27 & 0,26 & 0,27 & 0,28 & 0,21 & 0,25 & 0,18 \\
\hline $\mathrm{MnO}$ & 0,12 & 0,13 & 0,08 & 0,13 & 0,17 & 0,12 & 0,16 & 0,13 & 0,14 & 0,13 & 0,20 & 0,13 \\
\hline LOI & 2,4 & 1,8 & 1,1 & 2,4 & 2,6 & 1,6 & 1,3 & 1,6 & 1,4 & 2,0 & 2,8 & 3,7 \\
\hline SUMA & 99,70 & 99,68 & 99,65 & 99,76 & 99,74 & 99,76 & 99,72 & 99,67 & 99,67 & 99,79 & 99,39 & 99,70 \\
\hline La & 21,7 & 17,8 & 11,9 & 5,3 & 28,1 & 13,6 & 13,3 & 16,4 & 18,1 & 18,1 & 18,1 & 9,4 \\
\hline $\mathrm{Ce}$ & 38,1 & 33,3 & 21,0 & 8,3 & 37,4 & 25,9 & 24,7 & 32,2 & 34,1 & 29,6 & 27,9 & 16,7 \\
\hline Pr & 4,86 & 4,27 & 2,71 & 1,53 & 4,80 & 3,37 & 3,27 & 4,09 & 4,39 & 3,75 & 3,45 & 2,09 \\
\hline Nd & 20,5 & 17,0 & 11,8 & 8,5 & 21,0 & 14,5 & 14,6 & 17,6 & 18,0 & 15,7 & 14,2 & 8,7 \\
\hline Sm & 3,88 & 3,67 & 2,52 & 2,54 & 3,84 & 3,12 & 3,23 & 3,65 & 3,83 & 3,45 & 3,37 & 1,90 \\
\hline $\mathbf{E u}$ & 1,13 & 1,08 & 0,77 & 1,79 & 1,20 & 1,00 & 0,99 & 1,07 & 1,17 & 0,97 & 1,39 & 0,71 \\
\hline Gd & 3,50 & 3,12 & 1,98 & 2,99 & 3,48 & 2,85 & 3,06 & 3,01 & 3,44 & 3,30 & 3,23 & 1,96 \\
\hline $\mathbf{T b}$ & 0,45 & 0,43 & 0,22 & 0,47 & 0,46 & 0,36 & 0,44 & 0,40 & 0,45 & 0,47 & 0,47 & 0,29 \\
\hline Dy & 2,33 & 2,47 & 1,14 & 2,81 & 2,62 & 1,88 & 2,57 & 2,38 & 2,68 & 2,89 & 3,02 & 1,85 \\
\hline Ho & 0,46 & 0,47 & 0,20 & 0,63 & 0,48 & 0,36 & 0,53 & 0,42 & 0,48 & 0,56 & 0,58 & 0,40 \\
\hline Er & 1,29 & 1,38 & 0,49 & 1,93 & 1,30 & 0,87 & 1,45 & 1,17 & 1,38 & 1,50 & 1,78 & 1,04 \\
\hline Tm & 0,21 & 0,18 & 0,08 & 0,28 & 0,21 & 0,11 & 0,22 & 0,19 & 0,21 & 0,23 & 0,26 & 0,17 \\
\hline $\mathbf{Y b}$ & 1,36 & 1,28 & 0,51 & 1,66 & 1,24 & 0,69 & 1,44 & 1,27 & 1,39 & 1,48 & 1,77 & 1,24 \\
\hline Lu & 0,19 & 0,20 & 0,08 & 0,26 & 0,21 & 0,11 & 0,24 & 0,20 & 0,20 & 0,23 & 0,26 & 0,18 \\
\hline Cs & 0.1 & 1,2 & 0,5 & 0,4 & 0,3 & 0,5 & 0,5 & 0,9 & 0,5 & 1,5 & 0,4 & 1,4 \\
\hline $\mathbf{R b}$ & 37,5 & 46,1 & 23,9 & 5,5 & 41,0 & 32,1 & 33,9 & 48,5 & 52,2 & 57,6 & 42,2 & 45,4 \\
\hline $\mathrm{Ba}$ & 1092 & 1170 & 1304 & 258 & 720 & 799 & 992 & 1047 & 1118 & 673 & 3706 & 1306 \\
\hline Th & 3,5 & 3,0 & 1,6 & 0,9 & 3,1 & 2,2 & 2,1 & 2,7 & 3,1 & 4,0 & 3,1 & 2,1 \\
\hline $\mathbf{U}$ & 1,5 & 1,3 & 0,8 & 0,4 & 1,5 & 1,0 & 1,0 & 1,2 & 1,7 & 1,2 & 1,3 & 0,9 \\
\hline $\mathrm{Nb}$ & 5,9 & 5,2 & 2,3 & 2,1 & 5,8 & 4,1 & 5,0 & 6,0 & 6,4 & 5,7 & 3,4 & 3,1 \\
\hline Ta & 0,4 & 0,3 & 0,1 & 0,1 & 0,4 & 0,3 & 0,3 & 0,4 & 0,4 & 0,4 & 0,2 & 0,2 \\
\hline $\mathbf{P b}$ & 2,5 & 1,6 & 1,5 & 7,0 & 8,9 & 1,2 & 2,7 & 1,4 & 1,7 & 1,6 & 20,8 & 8,0 \\
\hline $\mathrm{Sr}$ & 934,0 & 942,8 & 1079,0 & 701,1 & 927,3 & 731,2 & 774,8 & 1065,1 & 926,0 & 597,8 & 995,9 & 714,5 \\
\hline $\mathbf{Y}$ & 13,8 & 13,8 & 5,8 & 18,4 & 14,9 & 9,7 & 16,6 & 13,1 & 15,1 & 17,1 & 17,0 & 10,5 \\
\hline $\mathrm{Sr} / \mathbf{Y}$ & 67,7 & 68,3 & 186,0 & 38,1 & 62,2 & 75,4 & 46,7 & 81,3 & 61,3 & 35,0 & 58,6 & 68,0 \\
\hline Co & 7,7 & 9,2 & 11,1 & 14,8 & 10,4 & 8,9 & 10,8 & 12,4 & 13,9 & 5,6 & 4,2 & 7,2 \\
\hline $\mathrm{Cu}$ & 8,3 & 22,4 & 57,4 & 54,1 & 12,6 & 13,1 & 14,9 & 36,0 & 43,1 & 3,8 & 17,0 & 3,5 \\
\hline $\mathrm{Zn}$ & 85 & 86 & 55 & 85 & 117 & 87 & 73 & 118 & 85 & 80 & 114 & 96 \\
\hline $\mathrm{Ni}$ & 2,6 & 3,7 & 6,4 & 12,8 & 4,9 & 4,1 & 4,6 & 5,1 & 5,4 & 1,1 & 5,8 & 0,8 \\
\hline $\mathbf{A u}$ & $<0.5$ & $<0.5$ & 0,9 & 5,4 & $<0.5$ & $<0.5$ & $<0.5$ & $<0.5$ & $<0.5$ & $<0.5$ & 0,7 & $<0.5$ \\
\hline
\end{tabular}


TABLA 2. Datos isotópicos de Sr-Nd

Concentración Inicial Actual

\begin{tabular}{lccccccccccc}
\hline Muestra & $\mathbf{8 7 S r} / \mathbf{8 6 S r}$ & $(+/-)$ & $\mathbf{1 4 3 N d} / \mathbf{1 4 4 N d}$ & $(+/-)$ & $\mathbf{1 4 7} \mathbf{S m} / \mathbf{1 4 4 N d}$ & edad $(\mathrm{Ma})$ & $\mathrm{Sm}$ & $\mathrm{Nd}$ & $\boldsymbol{E}$ Nd & $\boldsymbol{E}$ Nd & $(+/-)$ \\
\hline TB6 WRB1 & 0,704293 & \pm 4 & 0,512849 & \pm 10 & $\mathbf{0 , 1 3 0 7}$ & 9 & 3,73 & 17,3 & $\mathbf{4 , 3}$ & 4,3 & $\pm 0,2$ \\
TB9 WRB1 & 0,704201 & \pm 5 & 0,512893 & \pm 9 & $\mathbf{0 , 1 2 7 7}$ & 9 & 2,55 & 13,1 & $\mathbf{5 , 2}$ & 5,1 & $\pm 0,2$ \\
TB17 WRB1 & 0,704076 & \pm 4 & 0,512907 & \pm 9 & $\mathbf{0 , 1 3 8 1}$ & 9 & 3,77 & 16,5 & $\mathbf{5 , 5}$ & 5,4 & $\pm 0,2$ \\
TB22 WRB1 & 0,704219 & \pm 4 & 0,512872 & \pm 9 & $\mathbf{0 , 1 2 8 1}$ & 9 & 4,01 & 18,9 & $\mathbf{4 , 8}$ & 4,7 & $\pm 0,2$ \\
\hline
\end{tabular}

Los elementos traza muestran en un diagrama multielemental normalizado con respecto a condritos (FIGURA 5A), elementos litófilos de radio iónico grande LILE (Rb, Sr, Ba, U, Pb, Cs, Th) tienen un comportamiento similar con excepción de la muestra de lava con composición basáltica, la cual presenta un enriquecimiento menor con respecto a las otras muestras, no obstante los elementos como Sr, U y $\mathrm{Pb}$ tienen un patrón similar. En los elementos de alto

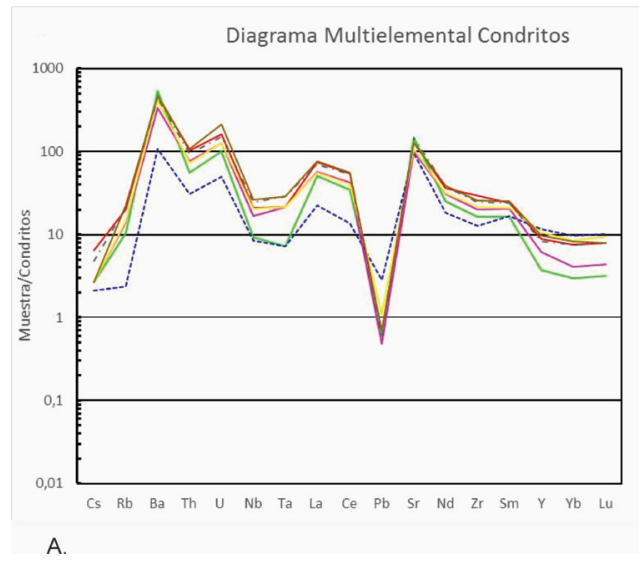

potencial iónico, $\operatorname{HFSE}(\mathrm{Zr}, \mathrm{Nb}, \mathrm{Ta})$, la tendencia del empobrecimiento es muy mezclado y no hay un patrón definido en todas las muestras. En un diagrama de $\mathrm{Sr} / \mathrm{Y}$ vs Y (FIGURA 5B) la mayoría de las muestras se localizan en el campo de rocas con afinidad adaquítica (Castillo, 2006), con excepción de la muestra de lava de composición basáltica (TB-10) y la muestra del cuerpo sub-volcánico más sur del área estudiada (TB26, FIGURA 1).

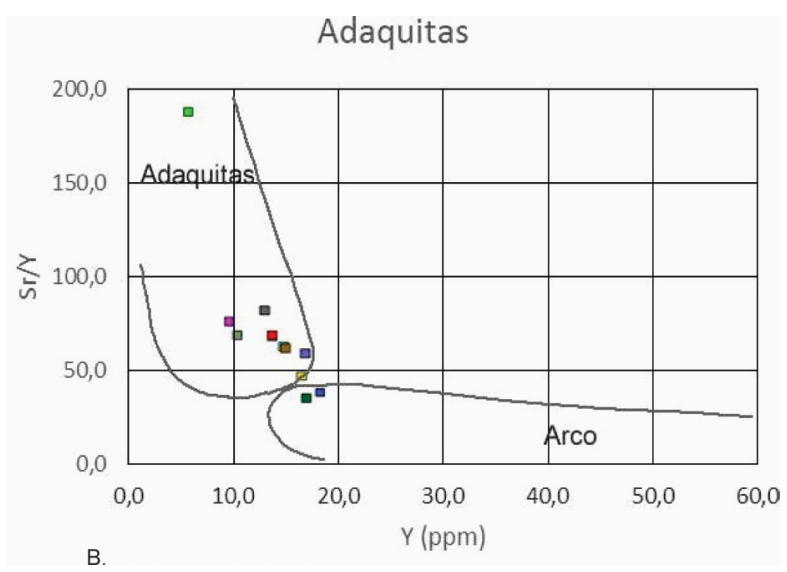

FIGURA 5. A. Diagrama multi-elemental normalizado con valores de condritos de Sun and McDonough (1989) mostrando una firma geoquímica típica de subducción. B. Diagrama Sr/Y vs Y para discriminar los campos de adaquitas y calcoalcalinas normales de arco, campos tomados de Castillo et al. (1999). Los colores de las muestras igual a la numeración de la leyenda de la FIGURA 4.

El diagrama multi-elemental de elementos de tierras raras, REE, normalizado a condritos (Sun and McDonough, 1989) muestra un grupo dominante de muestras con enriquecimiento en las tierras raras livianas, LREE (La, Ce, Pr, Nd Pm), con excepción de la muestra de lava de composición basáltica (FIGURA 6). En las tierras raras medianas, MREE (Sm, Eu, Gd, $\mathrm{Tb}$, Dy) la mayoría de las muestras presentan igual tendencia de empobrecimiento y ausencia de una anomalía de Eu, con excepción de la muestras TB-
10 que tienen un patrón contrario de enriquecimiento muy distinto al resto de las muestras, con una anomalía positiva de Eu muy marcada. El patrón de las tierras raras pesadas, HREE (Ho, Er, Tm, Yb, Lu) en las muestras tiene una tendencia plana regular constante, donde solo dos de las muestras de los cuerpos subvolcánicos (TB-9 y TB-17) tienen una relaciones diferentes de MREE/HREE (Gd/Yb) >3,9 y de LREE/ HREE $(\mathrm{La} / \mathrm{Yb})>19,7$ con respecto a las otras muestras (FIGURA 7). 


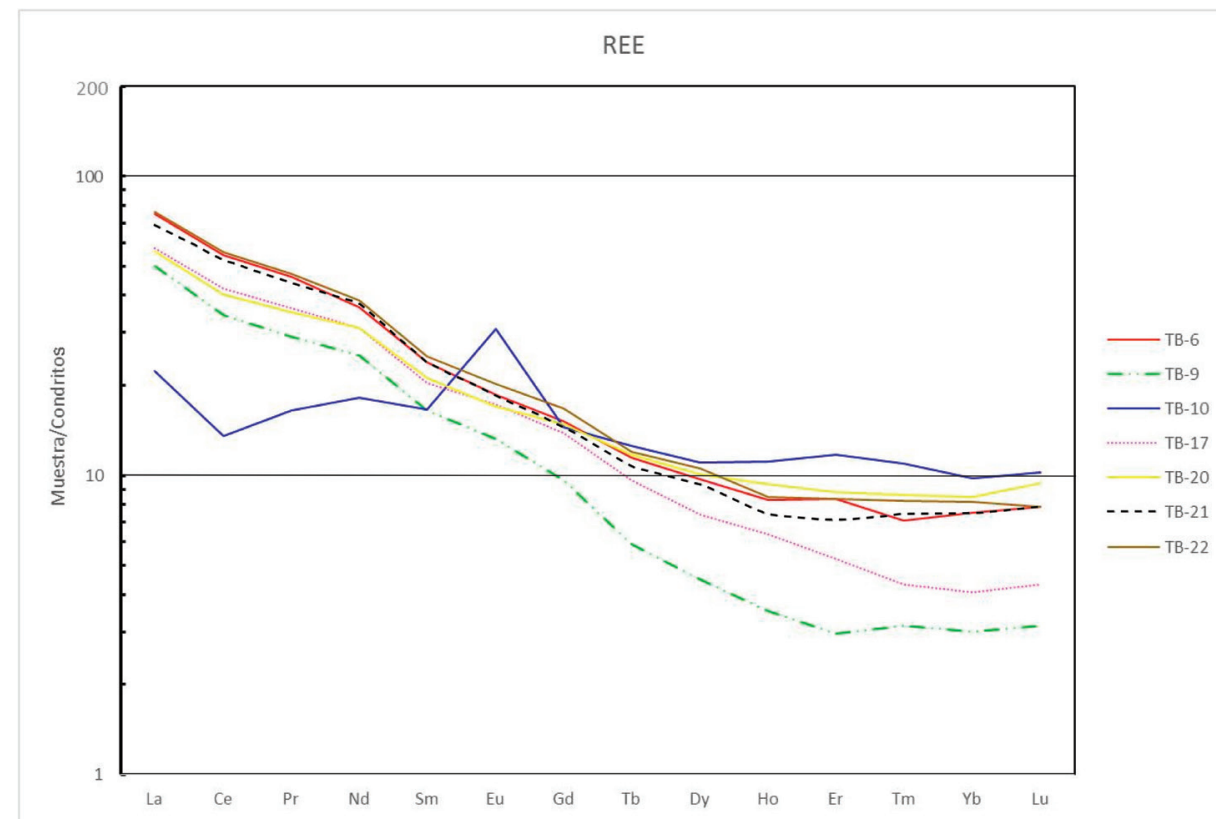

FIGURA 6. Diagrama multi-elemental de tierras raras (REE) normalizado a Condritos (Sun and McDonough, 1989), en general las muestras están enriquecidas en LREE y empobrecidas en HREE, Nótese el comportamiento diferente en los LREE de la muestra TB-10, basalto del Miembro Inferior de la Formación Combia con respecto a las otras muestras. Colores de las muestras igual que en la leyenda de la FIGURA 4.
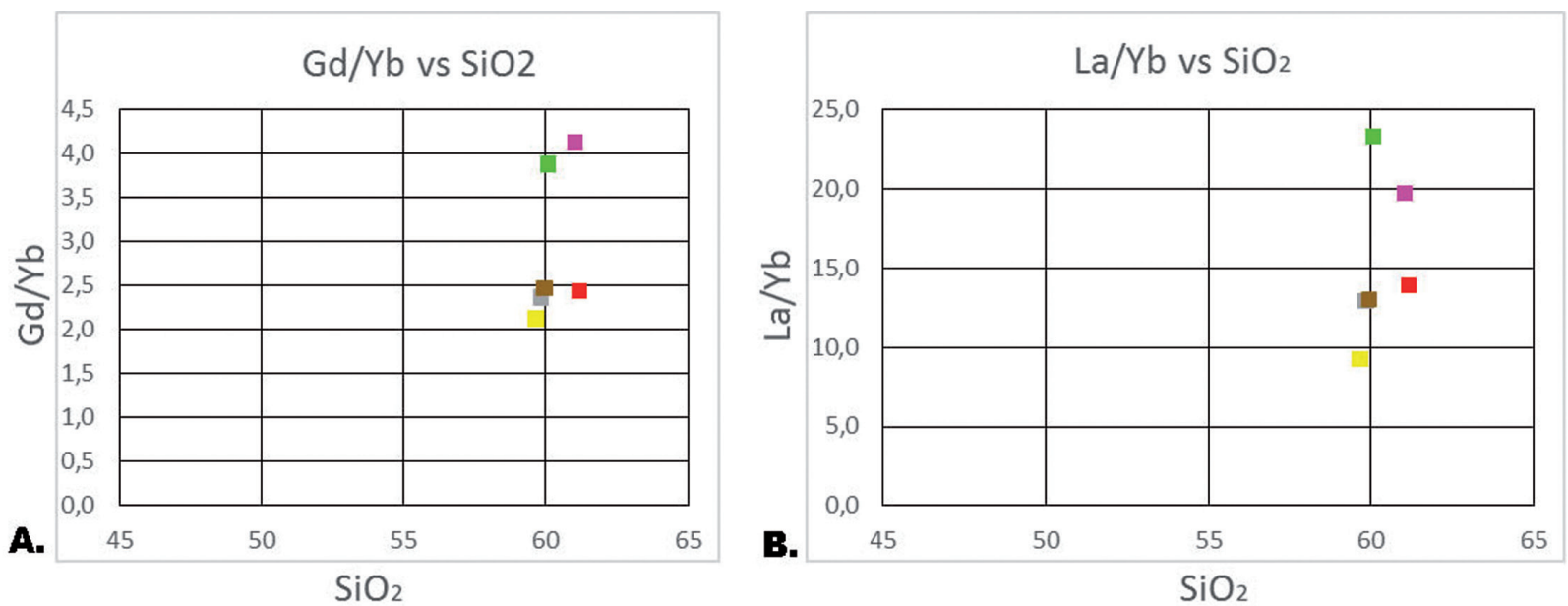

FIGURA 7. A. Proporciones de MREE/HREE $(\mathrm{Gd} / \mathrm{Yb})$ vs $\mathrm{SiO}_{2}$. B. Proporciones de LREE/HREE (La/Yb) vs $\mathrm{SiO}$. Las proporciones en las muestras TB-9 y TB-17 (localizadas en la parte superior) se podrían tomar como indicios de una proveniencia magmática asociado a la placa subducente. Colores de las muestras igual que en la leyenda de la FIGURA 4.

\section{Relaciones Isotópicas}

Las relaciones isotópicas de ${ }^{87} \mathrm{Sr} /{ }^{86} \mathrm{Sr}$ y ${ }^{143} \mathrm{Nd} /{ }^{144} \mathrm{Nd}$ de las muestras seleccionadas (TABLA 2 y FIGURA 1) muestran valores muy cercanos: ${ }^{87} \mathrm{Sr} /{ }^{86} \mathrm{Sr}$ entre 0,704201 $\pm 0,00004$ y $0,704293 \pm 0,00004$ y ${ }^{143} \mathrm{Nd} /{ }^{144} \mathrm{Nd}$ entre $0,512849 \pm 0,00010$ y $0,512907 \pm 0,00009$ (FIGURA 8). Estos valores se traslapan con los datos isotópicos del Miembro Inferior de la Formación Combia y otros cuerpos sub-volcánicos localizados al norte de nuestra área (Leal-Mejía, 2011), del Stock de Marmato (Tassinari et al., 2008) localizados al suroeste de la subcuenca de Amagá y de las adaquitas del prospecto La Colosa (Gil-Rodríguez, 2010) localizada en la parte media de la Cordillera Central. Estos valores presentan una firma que los ubica dentro del campo del arreglo mantélico. 


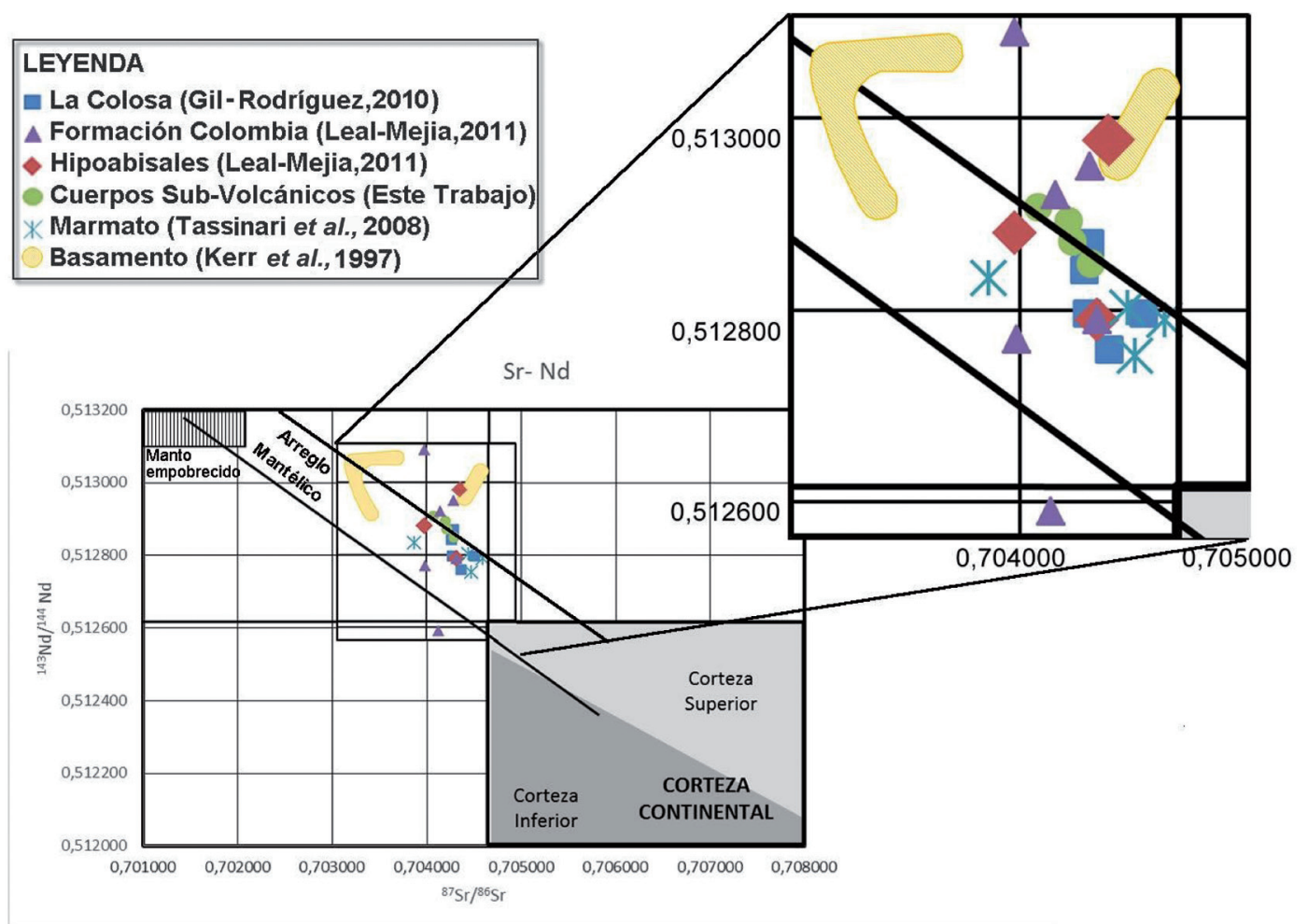

FIGURA 8. Las relaciones isotópicas de ${ }^{143} \mathrm{Nd} /{ }^{144} \mathrm{Nd}$ y ${ }^{87} \mathrm{Sr} /{ }^{86} \mathrm{Sr}$ de las muestras de este trabajo (círculos verdes), comparadas con datos isotópicos de Gil-Rodríguez (2010), Leal-Mejía (2011) y Tassinari et al. (2008). La mayoría de las muestras están localizadas en el arreglo mantélico o cerca de su límite. Para comparación se incluyen los datos de Kerr et al. (1997) como probable basamento del área.

\section{DISCUSIÓN}

Los depósitos volcaniclásticos de la Formación Combia están descritos principalmente desde el punto de vista estratigráfico (e.g. Grosse, 1926; González, 1980; López et al., 2006), y de manera general su geoquímica (e.g. Jaramillo, 1976 ; Ordóñez y Pimentel, 2001; LealMejía, 2011), pero no se ha discutido cual podría haber sido el origen y los tipos de magmas de esta unidad estratigráfica. Este trabajo explora con base en datos geoquímicos e isotópicos como fue el volcanismo del Miembro Inferior de la Formación Combia en el sector sur de la subcuenca de Amaga.

Las edades establecidas para la Formación Combia la ubican en el Mioceno tardío, con base principalmente en las dataciones de los cuerpos sub-volcánicos que la intruyen; 9,1 Ma del cuerpo sub-volcánico El Cangrejo en el suroeste de Antioquia (Restrepo et al., 1981); edades de $8 \mathrm{Ma}$ (M. Aránzazu, comunicación oral, 2013) en el área de La Cumbre (estación TB-32 en la FIGURA 1, Quinchía, departamento de Risaralda); y las edades del Stock de Marmato (FIGURA 1, estación TB-4), Ar-Ar

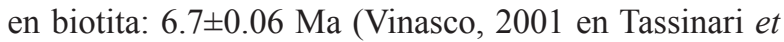

al., 2008), y K/Ar en hornblenda: 6,3 $\pm 0,7 \mathrm{Ma}$ (Restrepo et al., 1981). Otras edades reportadas están basadas en dataciones radiométricas, principalmente $\mathrm{K} / \mathrm{Ar}$ en roca total y huellas de fisión en circones, las cuales varían entre 10,6 $\pm 1,2$ y $6.3 \pm 0,7 \mathrm{Ma}$ (Ramírez et al., 2006) y entre $15 \pm 0,75$ y 6,3 $\pm 0,7 \mathrm{Ma}$ (López et al., 2006). La única datación radiométrica directa del Miembro Inferior en el suroeste de Antioquia es de una andesita, K-Ar en roca total: $c a$. 6,1 Ma (Leal-Mejía, 2011). La mayoría de edades marcan el enfriamiento de las rocas, por lo tanto para establecer los rangos de edad de este vulcanismo son necesarias edades $\mathrm{U} / \mathrm{Pb}$.

Debido al espesor de más de $1.000 \mathrm{~m}$ del Miembro Inferior en la sub-cuenca de Amagá, no existen datos geocronológicos que definan el inicio de este vulcanismo. Tampoco se tienen edades de los depósitos piroclásticos que afloran en el área de trabajo, que probablemente estarían relacionados con la explosión/ destrucción de algunos de los domos, domos-coladas, cuellos y espinas volcánicas que formaban los edificios del arco magmático del Mioceno tardío. Remanentes de parte de estos edificios son los cuerpos sub-volcánicos descritos en este trabajo (FIGURA 3). 
Los análisis químicos muestran como la mayoría de las muestras del Miembro Inferior de la Formación Combia y los cuerpos sub-volcánicos que la intruyen son parte de las series calco-alcalinas, andesitas y dacitas (FIGURA 4A) y solo la lava de composición basáltica (muestra TB-10) es parte de las series toleíticas (FIGURA 4B), similar a lo propuesto por Jaramillo (1976); Ordóñez y Pimentel (2001) y Leal-Mejía (2011). López et al. (2006), consideran las rocas con afinidad toleítica como la parte basal del Miembro Inferior, y localizan las rocas con afinidad calcoalcalina hacia el tope del miembro, pero sin incluir los cuerpos sub-volcánicos. Características como los patrones de elementos traza mostradas en la FIGURAS 5A y 6, las anomalías positivas de Cs y $\mathrm{Ba}$ y las negativas de $\mathrm{Nb}$ y $\mathrm{Ta}$, el empobrecimiento en $\mathrm{Pb}$, y los altos valores de las relaciones $\mathrm{Sr} / \mathrm{Nd}$ y Ba/ LREE, nos indican que las rocas del Miembro Inferior de la Formación Combia y los cuerpos sub-volcánicos están relacionados a un marco tectónico de subducción (cf. Leal-Mejía, 2011 y referencias incluidas). Esto sugiere la actividad de un arco magmático que podría correlacionarse con el denominado "vulcanismo tipo Combia” por Ramírez et al. (2006).

El mayor contraste con las áreas estudiadas en el departamento de Antioquia es que la mayoría de las muestras de andesitas y dacitas de los cuerpos subvolcánicos de nuestra área de trabajo son ricas en plagioclasa y anfíbol, y muestran una afinidad adaquítica (FIGURA 5B). Las características geoquímicas que sugieren esta afinidad y concuerdan en su mayoría con las propuestas por Castillo (2006) son: $\mathrm{SiO}_{2}>56 \%$, $\mathrm{Al}_{2} \mathrm{O}_{3}>15 \%, \mathrm{MgO}<3 \%, \mathrm{Na}_{2} \mathrm{O}$ entre 3,5 y $7,5 \%, \mathrm{~K}_{2} \mathrm{O} /$ $\mathrm{Na}_{2} \mathrm{O} \sim 0.42$, alto fraccionamiento de HREE, ausencia de anomalías de $\mathrm{Eu}, \mathrm{Yb} \leq 1,8 \mathrm{ppm}, \mathrm{Y}<18 \mathrm{ppm}$, altos contenidos de $\mathrm{Sr}(>400 \mathrm{ppm}), \mathrm{Sr} / \mathrm{Y}>20$ y La/Yb $>20$, y bajas relaciones de ${ }^{87} \mathrm{Sr} /{ }^{86} \mathrm{Sr} \leq 0,0704$ (FIGURAS $5 \mathrm{~A}$, 6 y 8$)$.

Existe una amplia controversia sobre el origen de los magmas adaquíticos (e.g. Chiaradia, 2015), por ejemplo los que se localizan al SE de Manizales, departamento de Caldas y que formaron parte del episodio constructivo del Ruiz Ancestral ( 1,8 Ma). De acuerdo con Toro-Toro et al. (2008) fueron derivados de la fusión parcial de una corteza oceánica subducida joven y caliente $(<20 \mathrm{Ma})$ de composición basáltica y con una leve participación de la cuña mantélica. Otros autores (e.g. Bourdon et al., 2002; Castillo, 2006) incluyen fenómenos de desgarre de la placa subducente, o subducción plana. Otras posibilidades mencionan fusión de la cuña mantélica por los fluidos de la placa subducente, o fusión parcial de corteza inferior máfica (Chiaradia et al., 2009). Pero algunos de estos mismos procesos petrogenéticos son invocados para explicar los patrones geoquímicos de los volcanes del sur de Colombia, cuyos productos en general no tienen afinidad adaquítica (e.g., Droux and Delaloye, 1996; Calvache and Williams, 1997; MarínCerón et al., 2010).

También hay que tener en cuenta el espesor de la corteza en el sector sur de la subcuenca de Amagá, 35 km (Vargas, 2014, comunicación escrita), ya que para Chiaradia (2015) las cortezas más gruesas, $>30 \mathrm{~km}$, constituyen el factor de primer orden en la generación de los magmas adaquíticos (con muy altos valores de $\mathrm{Sr} / \mathrm{Y}$, como los mostrados en la TABLA 1), más que los procesos mismos relacionados con las fuentes del magma. Este espesor no solo favorecería la evolución de estos magmas a niveles corticales promedios más profundos, sino que permitiría una diferenciación magmática más extensiva a estos niveles, resultando en contenidos progresivamente más bajos de $\mathrm{MgO}$ en condiciones de presión promedio más altas donde los minerales que contienen Y (i.e. anfíbol \pm granate) son estables y la plagioclasa que contiene Sr no sería estable, resultando en valores más altos de $\mathrm{Sr} / \mathrm{Y}$.

En este trabajo se propone que las firmas adaquíticas encontradas en el sur de la subcuenca de Amagá podrían estar relacionadas con fundidos derivados del manto como lo sugieren la homogeneidad isotópica de $\mathrm{Sr}-\mathrm{Nd}$ y las muy bajas composiciones isotópicas radiogénicas de Sr, tanto para las muestras de este trabajo como para las del Stock de Marmato (Tassinari et al., 2008) y de los cuerpos hipoabisales de la Formación Combia (Leal- Mejía, 2011; ver FIGURA 8). Para Tassinari et al. (2008) estas relaciones isotópicas sugieren un magma derivado del manto como principal fuente tanto para el magmatismo toleítico y calcoalcalino de la Formación Combia, y discuten que no puede ser descartada una contribución magmática menor resultante de la fusión de la corteza continental inferior.

La concentraciones de HFSE y las proporciones de MREE/HREE $(\mathrm{Gd} / \mathrm{Yb})$ y de LREE/HREE $(\mathrm{La} / \mathrm{Yb})$ (FIGURAS 7A y B), de las muestras de los cuerpos sub-volcánicos (TB-9 y TB-17) se podrían tomar como indicios de una proveniencia magmática asociado a la placa subducente; ésto debido a que el carácter insoluble de los elementos HFSE es muy debatido para los magmas adaquíticos. Por ejemplo, en la Zona Volcánica Norte en Ecuador, Bourdon et al. (2002) postulan la posibilidad de que puedan ser movilizados si los componentes de la subducción están en la forma de un fundido proveniente de la placa subducente o de su 
cubierta sedimentaria; isótopos de $\mathrm{Pb}$ confirmarían esta interpretación. La diferencia de comportamiento de las dos muestras de los cuerpos sub-volcánicos (FIGURA 7) también podría estar relacionada a una fuente metabasáltica de la placa Nazca subducida dentro del campo de estabilidad de granate \pm anfíbol ( $c f$. Gómez-Tuena et al., 2008), pero en la petrografía no se observó ninguna diferencia que permitiría discernir sobre este comportamiento diferente.

Para la zona de trabajo fue difícil comprobar de manera directa si hubo interacción con los sectores corticales medios e inferiores ya que no se encontraron xenolitos en las muestras que nos indiquen el tipo de material de la corteza. Sin embargo, parece no existir la posibilidad de una asimilación o mezcla con materiales corticales máficos, si tomamos en cuenta datos isotópicos de rocas similares al basamento de la parte sur de la subcuenca de Amagá, que se localizan al occidente de Colombia (Kerr et al., 1997): ${ }^{87} \mathrm{Sr} /{ }^{86} \mathrm{Sr}$ entre 0,70324 y $0,703686, \mathrm{y}{ }^{143} \mathrm{Nd} /{ }^{144} \mathrm{Nd}$ entre 0,512909 y 0,513063 (FIGURA 8), los cuales no son isotópicamente comparables a los fundidos derivados del manto; aunque se necesitarían otros estudios multiisotópicos para discernir la probable asimilación en la corteza baja, ya que de acuerdo con lo propuesto por Chiaradia (2015), el espesor de la corteza de 35 $\mathrm{km}$ en el sector sureste de la subcuenca de Amagá, podría haber permitido la generación de los magmas adaquíticos en niveles corticales profundos.

Los depósitos y los cuerpos volcánicos del Mioceno tardío, descritos en este trabajo, forman parte de la firma relacionada con la colisión tectónica de Panamá contra la esquina noroeste de Suramérica (Sierra and Marín, 2011) que comenzó hace 25-23 Ma (Farris et al., 2010). Resultado de esta colisión, es la intensa actividad magmática que probablemente empezó primero en el norte de Ecuador hace $c a$. $21 \mathrm{Ma}$ (Schütte, 2010), continuó en el sur de Colombia hace $c a$. $17 \mathrm{Ma}$ (Leal-Mejía, 2011) y luego migró al centro y norte de Colombia hace ca. $12 \mathrm{Ma}$ (Ramírez et al., 2006; López et al., 2006), terminando su actividad hace $6 \mathrm{Ma}$ (Restrepo et al., 1981; Tassinari et al., 2008; Leal-Mejía, 2011). La no continuidad de la actividad volcánica después de $6 \mathrm{Ma}$ en el sector sur de la subcuenca de Amagá podría estar relacionada con el "Caldas tear" que comenzó su propagación hace $c a$. $10 \mathrm{Ma}$ (Vargas and Mann, 2013). Este desagarre de la placa subducente separa una zona de subducción normal $\left(30-40^{\circ}\right)$ en el centro y sur de Colombia de una con subducción de ángulo bajo (20$30^{\circ}$ ) en la zona adyacente a Panamá y el mar Caribe ( $c f$. Vargas and Mann, 2013).

\section{CONCLUSIONES}

El vulcanismo del Mioceno tardío en la parte sur de la subcuenca de Amagá está asociado al Miembro Inferior de La Formación Combia. Los cuerpos sub-volcánicos porfiríticos que la intruyen, y que afloran en los departamentos de Caldas y Risaralda, están relacionados a un marco tectónico de subducción caracterizado por típicas anomalías positivas de Cs y Ba y las negativas de $\mathrm{Nb}$ y $\mathrm{Ta}$, el empobrecimiento en $\mathrm{Pb}$, y altos valores de las relaciones $\mathrm{Sr} / \mathrm{Nd}$ y $\mathrm{Ba} / \mathrm{LREE}$, que se correlacionan con el denominado "vulcanismo tipo Combia". Las muestras también presentan una firma adaquítica definida por el contenido de $\mathrm{SiO}_{2}>56 \%, \mathrm{Al}_{2} \mathrm{O}_{3}>15 \%$, $\mathrm{MgO}<3 \%, \mathrm{Na}_{2} \mathrm{O}$ entre 3,5 y 7,5\%, $\mathrm{Yb} \leq 1,8 \mathrm{ppm}, \mathrm{Y}$ $<18 \mathrm{ppm}, \mathrm{y} \mathrm{Sr}>400 \mathrm{ppm}$, el alto fraccionamiento de HREE, la ausencia de anomalías de Eu y las relaciones de $\mathrm{K}_{2} \mathrm{O} / \mathrm{Na} 2 \mathrm{O} \sim 0,42, \mathrm{Sr} / \mathrm{Y}>20, \mathrm{La} / \mathrm{Yb}>20, \mathrm{y}^{87} \mathrm{Sr} /{ }^{86} \mathrm{Sr}$ $\leq 0,0704$. Dos grupos son definidos por elementos de tierras raras, el primero por dos de las muestras de los cuerpos sub-volcánicos (TB-9 y TB-17) que tienen unas relaciones más altas de MREE/HREE $(\mathrm{Gd} / \mathrm{Yb})>3,9$ y de LREE/HREE $(\mathrm{La} / \mathrm{Yb})>19,7$ en comparación con el segundo grupo conformado por las muestras restantes.

Para las adaquitas encontradas en el sur de la subcuenca de Amagá, se sugiere que podrían estar relacionadas con fundidos derivados del manto, previamente metasomatizados por el componente de subducción, debido a la homogeneidad isotópica de $\mathrm{Sr}-\mathrm{Nd}$ y las muy bajas composiciones isotópicas radiogénicas de $\mathrm{Sr}$, tanto para las muestras de este trabajo como para las del Stock de Marmato y de los cuerpos hipoabisales de la Formación Combia. Sin embargo, no se descarta la posibilidad de tener también alguna mezcla con fundidos de la placa subducente debido a las diferentes relaciones de MREE/HREE y LREE/HREE, que separan las muestras en dos grupos. El espesor de la corteza de 35 $\mathrm{km}$ en el sector sureste de la subcuenca de Amagá es un factor determinante en la generación de los magmas adaquíticos en niveles corticales profundos.

Las rocas y depósitos aquí descritos corresponden al arco magmático calcoalcalino de edad Mioceno del norte de los Andes. Sin embargo, aún se desconoce cuándo comenzó la actividad volcánica, debido al gran espesor de la unidad en el sur de la subcuenca de Amagá. El vulcanismo bimodal, toleítico y calcoalcalino para el Miembro Inferior de la Formación Combia necesita más investigación, es necesario precisar la edad del volcanismo basáltico toleítico, y definir si está localizado sólo en la base de la unidad; un mayor detalle en su química también ayudaría a precisar su origen. De 
acuerdo con resultados de la caracterización química e isotópica presentados en este artículo, el Miembro Inferior de la Formación Combia debería incluir una suite de cuerpos sub-volcánicos adaquíticos como parte de esta unidad, por su estrecha relación estratigráfica y vulcanológica.

\section{AGRADECIMIENTOS}

Los autores agradecen al Dr. Jeff Vervoort, Washington State University por los análisis isotópicos $\mathrm{Sr}-\mathrm{Nd}$, a la Vicerrectoría de Investigaciones y al departamento de Ciencias Geológicas de la Universidad de Caldas por el patrocinio del proyecto de investigación; y a los geólogos H.F. Murcia (The Auckland University), S.M. Kay (Cornell University) y C. Vargas (Universidad Nacional de Colombia) por los comentarios y sugerencias sobre las primeras versiones de este artículo. A E. Gazel (Geosciences, Virginia Tech) y a un revisor anónimo por los comentarios y las discusiones sobre la estructura del artículo y el origen de las adaquitas.

\section{REFERENCIAS}

Álvarez, J.A. 1971. Informe preliminar sobre geoquímica de la Cordillera Occidental. INGEOMINAS, Medellín, Informe interno, $8 \mathrm{p}$.

Bourdon, E., Eissen, J.P., Monzier, M., Robin, C., Martin, H., Cotton, J., and Hall, M.L. 2002. Adakitelike lavas from Antisana volcano (Ecuador): evidence for slab melt metasomatism beneath the Andean northern volcanic zone. Journal of Petrology, 43(2): 199-217.

Calvache, M.L., and Williams, S. 1997. Geochemistry and petrology of the Galeras Volcanic Complex, Colombia. Journal of Volcanology and Geothermal Research, 77: 21-38.

Castillo, P.R. 2006. An overview of adakite petrogenesis. Chinese Science Bulletin, 51(3): 257-268.

Castillo, P.R., Janney, P.E., and Solidum, R.U. 1999. Petrology and geochemistry of Camiguin Island, southern Philippines: insights to the source of adakites and other lavas in a complex arc setting. Contributions to Mineralogy and Petrology, 134(1): 33-51.

Cediel, F., Leal-Mejía, H., Shaw, R.P., Melgarejo, J.C., and Restrepo-Pace, P.A. 2011. Regional geology of Colombia (Vol. 1). In: Cediel, F. (Ed.), Petroleum geology of Colombia, ANH- Universidad EAFIT, 225p.
Chiaradia, M. 2015. Crustal thickness control on $\mathrm{Sr} / \mathrm{Y}$ signatures of recent arc magmas: an Earth scale perspective. Scientific Report 5, 8115; DOI: 10.1038/ srep08115.

Chiaradia, M., Müntener, O., Beate, B., and Fontignie, D. 2009. Adakite-like volcanism of Ecuador: lower crust magmatic evolution and recycling. Contributions to Mineralogy and Petrology, 158:563-588.

Droux, A. and Delaloye, M. 1996. Petrography and geochemistry of Plio-Quaternary calc-alkaline volcanoes of southwestern Colombia. Journal of South American Earth Sciences 9: 27-41.

Farris, D.W., Jaramillo, C., Bayona, Restrepo-Moreno, S.A., Montes, C., Cardona, A., Mora, A., Speakman, R.J., Glascock, M.D., and Valencia, V. 2010. Fracturing of the Panamanian Isthmus during initial collision with South America. Geology 39: 1007-1010.

Gil-Rodríguez, J. 2010, Igneous petrology of the Colosa gold-rich porphyry system (Tolima, Colombia), PSM/ EG thesis, Tucson (Arizona), USA, University of Arizona, 35p.

Gill, J.B. 1981. Orogenic andesites and plate tectonics. Springer-Verlag Berlin, 390p.

Gómez, J., Nivia, A., Montes, N.E., Jiménez, D.M., Sepúlveda, J., Gaona, T., Osorio, J.A., Diederix, H., Mora, M., y Velásquez, M.E., (compiladores). 2007. Atlas geológico de Colombia. Escala 1:500.000. INGEOMINAS, 26 planchas. Bogotá.

Gómez-Tuena, A., Langmuir, C.H., Goldstein, S.L., Straub, S.M., and Ortega-Gutiérrez, F. 2007. Geochemical evidence for slab melting in the Trans-MexicanVolcanic Belt. Journal of Petrology, 48(3): 537-562.

González, H. 1980. Geología de las planchas 167 (Sonsón) y 168 (Salamina). Boletín Geológico, 23: 174p.

Grosse, E. 1926. El Terciario carbonífero de Antioquia. Berlín: Dietrich Reimer. E. Vohsen, 321p.

Irvine, T.N., and Baragar, W.R.A. 1971. A guide to the chemical classification of the common volcanic rocks. Canadian Journal of Earth Sciences, 8: 523-548.

Jaramillo, J.M. 1976. Volcanic rocks of the río Cauca valley, Colombia S.A. Thesis Degree of Master of Arts, Rice University, Houston. 
Kerr, A.C., Marriner, G.F., Tarney, J., Nivia, A., Saunders, A.D., Thirlwall, M.F., and Sinton, C.W. 1997. Cretaceous basaltic terranes in western Colombia: elemental, chronological and $\mathrm{Sr}-\mathrm{Nd}$ isotopic constraints on petrogenesis. Journal of Petrology 38(6): 677-702.

Leal-Mejía, H. 2011, Phanerozoic gold metallogeny in the Colombian Andes - A tectono-magmatic approach. Ph.D. thesis, Barcelona (Catalonia), Spain, University of Barcelona, 1.000p.

LeBas, M.J., Lemaitre, R.W., Streckeisen, A., and Zanettin, B. 1986. A chemical classification of volcanic rocks based on total alkali silica diagram. Journal of Petrology 27(3): 745-750.

López, A., Sierra, G.M., y Ramírez, D. 2006. Vulcanismo neógeno en el suroccidente antioqueño y sus implicaciones tectónicas. Boletín de Ciencias de la Tierra, 19: 27-42.

Marín-Cerón, M.I., Moriguti, T., Makishima, A., and Nakamura, E. 2010. Slab decarbonation and $\mathrm{CO} 2$ recycling in the southwestern Colombian volcanic arc. Geochimica et Cosmochimica Acta 74: 1104-1121.

Moreno, M., Gómez, A. de J., y Toro, L.M. 2008. Proveniencia del material clástico del Complejo Quebradagrande y su relación con los complejos estructurales adyacentes. Boletín de Ciencias de la Tierra, 22: 27-47..

Murcia, H.F., Borrero, C.A., Pardo, N., Alvarado, G., Arnosio, M., y Scolamacchia, T. 2013. Depósitos volcaniclásticos: términos y conceptos para una clasificación en español. Revista Geológica de América Central, 48:15-39.

Ordóñez, O., y Pimentel, M.M., 2001. Geoquímica isotópica del magmatismo reciente $(<11 \mathrm{Ma})$ en los Andes Colombianos. CD Memorias Congreso Colombiano de Geología, VII, Manizales.

Patarroyo, T.L. 2014. Vulcanismo de la parte media del Valle del Cauca (Colombia) a través de la petrografía. Trabajo de Grado de Geología. Universidad de Caldas. 86p.

Ramírez, D., López, A., Sierra, G.M., y Toro, G.E. 2006. Edad y proveniencia de las rocas volcánico sedimentarias de la Formación Combia en el suroccidente antioqueño, Colombia. Boletín de Ciencias de la Tierra, 19: 9-26.
Restrepo, J.J. Toussaint, J.F., y González, H. 1981. Edades mio-pliocenas del magmatismo asociado a la Formación Combia, departamentos de Antioquia y Caldas. Geología Norandina, 3: 21-26.

Schütte, Ph. 2010. Geochronology, geochemistry, and isotopic composition $(\mathrm{Sr}, \mathrm{Nd}, \mathrm{Pb})$ of tertiary porphyry systems in Ecuador. Thèse de doctorat: Univ. Genève, no. Sc. Pp. 4166, 203p.

Sierra, G., and Marín, M.I. 2011. Amagá, Cauca and Patía Basins (Vol. 2). In: Cediel, F. (Ed.), Petroleum geology of Colombia, ANH- Universidad EAFIT, 102p.

Sillitoe, R.H. 2008. Major gold deposits and belts of the North and South American Cordillera: distribution, tectonomagmatic settings, and metallogenic considerations. Special Paper, Economic Geology, 103: 663-687.

Sun, S.S., and McDonough, W.F. 1989. Chemical and isotopic systematics of oceanic basalts: implications for mantle composition and processes. In: Saunders A.D. and Norry, M.J. (eds.), Magmatism in the ocean basins. Geological Society London, Special Publications 42: 313-346.

Tassinari, C.C.G., Díaz, F., and Buenaventura, J. 2008. Age and sources of gold mineralization in the Marmato mining district, NW Colombia: a Miocene-Pliocene epizonal gold deposit. Ore Geology Reviews, 33: 505-518.

Tejada, M., Betancourt, J., Nivia, A., Weber, M., y Gómez, J. 2007. Cartografía geológica y caracterización geoquímica de la Formación Combia en los alrededores de Jericó y Pueblorrico, departamento de Antioquia Colombia. Memorias XI Congreso Colombiano de Geología. Bucaramanga.

Toro-Toro, L.M., Alvarán-Echeverri, M., y BorreroPeña, C.A. 2008. Rocas con afinidad adakítica al sureste de Manizales: rasgos petrogenéticos y geoquímicos. Boletín Geología, 30(2): 49-60.

Vargas, C.A., and Mann, P. 2013.Tearing and breaking off of subducted slabs as the result of collision of the Panama Arc-Indenter with northwestern South America. Bulletin of the Seismological Society of America, 103(3): 2025-2046.

Trabajo recibido: julio 10 de 2014

Trabajo aceptado: agosto 4 de 2015 